\title{
Consultoría en desarrollo organizacional y de negocios in situ
}

\author{
In Situ Organizational and Business Development Consulting
}

\section{Consultoria de desenvolvimento organizacional e de negócios in loco}

Daniel Matus Pérez ${ }^{* *}$

Universidad de Guanajuato, México

Cómo citar esta nota metodológica: Matus Pérez, D. (2019). Consultoría en desarrollo organizacional y de negocios in situ. Signos. Investigación en Sistemas de Gestión, 12(1), 23-30. DOI: https://doi.org/10.15332/24631140.5418

\section{RESUMEN}

La consultoría en desarrollo organizacional y de negocios es un área en constante crecimiento para la mejora del desempeño organizacional de las pymes. La gestión del cambio debe ser realizada de forma adecuada en la parte mecánica y dinámica, como en las diferentes etapas que la integran, las cuales, al omitirlas y no tomarlas en cuenta, se corre el riesgo de no generar el cambio esperado y desgastar a la organización con esfuerzos innecesarios, mal dirigidos o implementados. Conocer las etapas de un proceso de consultoría, entender los diferentes elementos que la constituye, así como la implementación de acuerdo con su etapa ayudará al adecuado establecimiento de los objetivos y a su cumplimiento. La presente nota metodológica busca ser un apoyo y referente para los consultores del área, aclarando que no es restrictiva ni excluyente, por lo que se puede complementar o se puede utilizar como apoyo a un proceso existente.

Artículo de investigación. Nota metodológica.

** Doctor en Administración, maestro en Desarrollo Organizacional, licenciado en Administración de Empresas, línea de investigación en Mejora del Desempeño Organizacional, Red para el Desarrollo de las Organizaciones (Redor), Universidad de Guanajuato, México. Correo electrónico: daniel@matusconsulting.com.mx Orcid: https://orcid.org/0000-0002-1203-0528 
Palabras clave: consultoría, pymes, desarrollo organizacional, desempeño organizacional, gestión del cambio, intervenciones.

\section{ABSTRACT}

Organizational and business development consulting is an area in constant growth for the improvement of the organizational performance of Small and Medium Enterprises (SMEs), change management must be carried out properly in the mechanical and dynamic part, as in the different stages that integrate it. By omitting and not taking these into account, you take the risk of not generating the expected change and weakening the organization with unnecessary, misdirected or implemented efforts. Knowing the stages of a consulting process, understanding the different elements that constitute these stages, as well as the implementation according to each stage, will help the proper objectives establishment and their fulfillment. This methodological note seeks to be a support and reference for the area consultants, clarifying that it is not restrictive nor exclusive, so it can be complemented or used as support for an existing process.

Keywords: consulting, SMEs, organizational development, organizational performance, change management, interventions.

\section{RESUMO}

A consultoria em desenvolvimento organizacional e de negócios é uma área que está evoluindo constantemente para melhorar o desempenho organizacional das PMEs (pequena e média empresa). A gestão de mudanças deve ser realizada adequadamente no âmbito mecânico e dinâmico, como nas suas diversas fases e se omitir ou ignorar essas etapas, existe 0 risco de não gerar a mudança esperada e desgastar à organização com esforços desnecessários, mal direcionados ou implementados. $\mathrm{O}$ fato de conhecer as diversas fases de um processo de consultoria, entender os diversos elementos que a compõem, bem como a implementação de acordo com sua fase auxiliará com o estabelecimento adequado dos objetivos e do seu cumprimento. Esta nota pretende ser um suporte e referência para os consultores da área, esclarecendo que não é limitativa e excludente, portanto, pode ser complementada ou utilizada como suporte a um processo existente.

Palavras-chave: consultoria, PMEs, desenvolvimento organizacional, desempenho organizacional, gestão da mudança, intervenções.

\section{CONTEXTUALIZACIÓN}

La consultoría de empresas comprende un campo de acción muy amplio y complejo, por lo que se debe tener claridad de los temas que se pueden atender y optimizar desde el ejercicio profesional de cada despacho de consultoría o de los profesionales independientes que ofrecen este servicio.

Revisando diversas fuentes y enriqueciéndolas con la experiencia del ejercicio profesional, se puede considerar que la consultoría es un método de asesoramiento profesional que ayuda a las organizaciones a través de modelos de intervención para establecer o cumplir sus objetivos, apoyando la solución de problemas, innovación de productos, servicios, modelos de negocio y gestión del conocimiento. Esta definición comprende e integra diferentes dimensiones de la organización abarcando las dimensiones operativas y administrativas (Matus, 2018). 
Es importante y necesario definir el campo de acción y el mercado que se pretende atender, no es lo mismo diseñar intervenciones para las grandes empresas que para las pymes. Las micro, pequeñas y medianas empresas (mipymes) son la espina dorsal de la economía mundial, su aportación es de suma importancia en diversos ámbitos, ya que representan más del $90 \%$ del total de empresas del mundo, generan entre el 60 y $70 \%$ de los empleos y, además, aportan el $50 \%$ del producto interno bruto (PIB) mundial, por ello son un factor clave para la reducción de la pobreza (ONU, 2018); de ahí la importancia de hacer énfasis en este segmento, además de que son las menos competitivas y las que tienen mayor probabilidad de fracaso.

La consultoría en desarrollo organizacional a pymes es recomendable realizarla in situ (en el sitio) para que el apoyo y conocimiento que requiere la empresa sea transferido por andamiaje (es la ayuda, información y guía que se recibe durante el aprendizaje) de parte de los consultores. Esto ayuda a la organización a desarrollar competencias con las que no cuenta y además tiene la ventaja de que se aplicarán a problemas específicos y en el momento que sucedan, logrando así transferencia de conocimiento y tecnología.

El modelo de intervención se integra de diversas etapas, cada una de las cuales es crucial para definir las actuaciones de la siguiente.

\section{CONTACTO INICIAL}

Se realizó mediante entrevista. Estos contactos iniciales generalmente se realizan por parte de los directivos o dueños de la organización; en ocasiones, algunos gerentes sienten la necesidad de iniciar un proceso de cambio o mejora. Se debe tener claridad desde un inicio que el proceso de cambio debe ser solicitado y avalado por la dirección general o por los dueños del negocio, en caso contrario, serácomplicado que el cambio prospere, sea aceptado por la organización o que las acciones emprendidas cuenten con el apoyo operativo, tecnológico o financiero que requieren. Un punto importante para generar el contacto inicial es que debe surgir de la empresa que busca el cambio hacia el despacho de consultoría; será complicado intentar iniciar un proceso de cambio por iniciativa o necesidad del consultor, lo primero que se debe tener es la necesidad de cambio desde la empresa.

En estas primeras entrevistas se bosqueja el alcance del proyecto, se debe entender cuál es la necesidad que motiva la intención de la gestión del cambio y se conceptualizan los objetivos; en la mayoría de las ocasiones, se requiere una mejora operativa o administrativa, son menos comunes las mejoras de cambio conductual (generalmente se contratan cursos o talleres que buscan solucionar los síntomas y no corrigen la causa que los genera), tampoco son comunes las solicitudes de gestión de cambio porque todo está bien y estático, esto se refiere a que la organización se encuentra en un estado de confort desde hace algún tiempo. Cuando la organización se instala en estos espacios de confort, se tiende a perder el impulso interior que llevó a la organización a crecer y mejorar sus resultados, a dar beneficios a sus clientes y colaboradores y a mejorar en productos y servicios. Esta etapa se caracteriza por la falta de innovación y falta de crecimiento, la operación se vuelve rutinaria, la organización carece de retos que le impulsen a mejorar.

En esta primera fase, se explican acciones iniciales a emprender y la forma de implementarlas, los tiempos, la forma de pago, también se detalla la experiencia del equipo que acompañará a la empresa para la gestión del cambio organizacional, además, se trata de sensibilizar al dueño y directores de la necesidad de que se involucren en el proyecto de forma integral, con pronunciamientos y en acciones, de lo contrario la organización se percatará de la falta de involucramiento y resistencia al proyecto. 


\section{Tiempo y alcance}

Existen intervenciones de diversos tipos, entre ellas, integrales y parciales; las primeras, comprenden e involucran a toda la organización (personal y áreas), mientras que la segunda implica un departamento o área específica. La necesidad de entender con claridad el alcance y tiempo, de acuerdo con lo expresado por los posibles clientes, radica en que a partir de esta comprensión se formulará la propuesta de trabajo, por lo que si el consultor no tiene la experiencia o capacidad para vislumbrar la raíz del problema o síntomas que se manifiestan en la organización, se corre el peligro de presentar una propuesta inadecuada en tiempo y alcance, lo que lleva a imprecisiones en costo y efectividad para el cumplimiento de los objetivos planteados.

Acciones e implementación. Las acciones inmediatas se enfocan en el contrato (requerimientos mínimos necesarios para iniciar el proyecto) y en el diagnóstico, que será la etapa próxima; a partir de estas acciones, se determinarán otras de mayor alcance y profundidad para impulsar los resultados del proyecto de intervención y que, además, son dinámicas.

\section{INICIO}

Contrato. Acordadas las formas de trabajo, el alcance, la duración del proyecto y los costos, el equipo que guiará y facilitará la gestión del cambio, se firma el contrato con los derechos y obligaciones para ambas partes. Es necesario hacer hincapié en que el contrato no debe ser un mecanismo de coacción hacia el cliente, sino al contrario, uno que busca otorgar toda la certeza al cliente de que recibirá lo que contrató, que su información será tratada y resguardada como confidencial, que el consultor no participará en negocios semejantes en algún lapso de tiempo, esto, por tranquilidad y respeto al negocio del cual se está compartiendo la información. En este mismo documento, se debe dejar claro que el cliente puede dar por terminado los servicios en el momento que desee; de igual forma, se debe dejar claramente estipulado por qué servicios debe pagar el cliente, en qué tiempo y la forma de estos.

Arranque. El kick off o inicio del proceso se da con una sesión informativa a los colaboradores de las diversas áreas en la cual se explica el tiempo y objetivo del proyecto, se presenta al equipo consultor y se detallan las acciones que se llevarán a cabo, la forma en que se observarán los procesos, la solicitud de información que será requerida y se disipan todas las dudas que pudieran generarse, además de que se inhibe la ansiedad que genera la desinformación en este tipo de procesos. En esta reunión, se aplica el primer instrumento de recolección de información.

Diagnóstico. Para conocer la situación de la empresa se emplean diversos métodos que brindan información que permita conocer y entender de primera fuente qué está aconteciendo en la organización, algunos de los instrumentos que facilitan el entendimiento de la organización son:

- Encuesta de clima organizacional

- Cuestionario estratégico a dueños

- Cuestionario a directivos y gerentes

- Entrevistas en profundidad con colaboradores y clientes

- Revisión de estados financieros

- Análisis de cultura organizacional

- Análisis de ciclos de vida de la organización

- Determinación de restricciones

- Análisis de modelo de negocio

- Descripción de la etapas y componentes del gobierno corporativo

- Revisión de presupuestos de ingresos y egresos

- Análisis de ventas 
- Conocimiento de alianzas estratégicas y posición en el mercado

- Documentación relevante a la estructura

- Cuestionario de descripción y análisis de puestos

- Análisis y clasificación de inventarios

- Observación de procesos

- Documentación legal sobre tenencia accionario

- Situación fiscal de la organización con autoridades

- Créditos

- Litigios

- Forma de contratación, de pago y terminación de las relaciones laborales

- Especialistas y asesores de la empresa

- Comportamiento de lealtad en clientes

Estos son algunos de los instrumentos, mecanismos, dinámicas y documentos para iniciar el diagnóstico de la organización. Es recomendable contar con la mayor información posible (solo la que aplique de acuerdo con el objetivo del proyecto) antes de tomar decisiones.

Conocimiento. De manera simultánea al diagnóstico, se debe conocer la organización, una parte importante son los documentos, pero otra igual de importante es vivir la organización, conocer la operación, entender cómo funciona desde adentro. En ocasiones, los consultores comenten el error de pensarse como "oráculos", y esto no es así; si bien un consultor debe contar con una amplia experiencia y tener bastos conocimientos del área y negocio que apoyará, ello no es suficiente, se debe conocer la empresa y su forma de operación, lo cual permitirá verificar si lo que se describió en el diagnóstico o la información que recibió va acorde a la realidad.

El diagnóstico no solo aporta una visión de las áreas de mejora, también permite conocer las fortalezas de la organización y los talentos por desarrollar.

Objetivo. Se debe analizar en profundidad la información documental y la operativa, esto permitirá establecer con precisión el objetivo del proyecto, suele suceder que en los contactos iniciales se habla de síntomas y resultados esperados, pero es después de recopilar la información que se puede detallar el alcance de la intervención.

\section{DESARROLLO}

Estrategia. Teniendo y entendiendo la información relevante y situación de la empresa, se determina la causa raíz de las situaciones que se desean modificar para cumplir los objetivos del proyecto. Se diseñan las estrategias por implementar, se verifica que sean acordes con la cultura que se tiene 0 que se busca, y se inicia la intervención operativa, que consiste en comenzar con los cambios. En esta parte del proyecto es cuando se diseñan las modificaciones a las estructuras, procesos y conductas alineadas al objetivo general y a la cultura de la organización (figura 1). El diseño de las estrategias debe seguir necesariamente ese orden, en caso contrario, será complejo intentar modificar situaciones aisladas. A partir de esta definición es que se tiene claridad de los recursos humanos, financieros y tecnológicos que se requieren para operar el cambio y también la capacidad de la organización para proveerlos o generarlos.

Figura 1. Niveles de desarrollo de estrategia

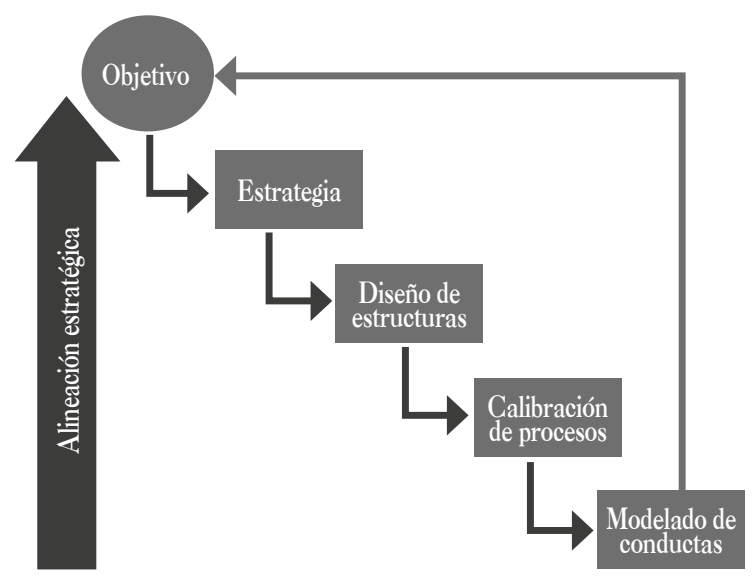

Fuente: elaboración propia. 
Implementación. La parte dinámica del proyecto se realiza a partir de esta etapa. Antes de iniciar cualquier acción (por sencilla que esta sea), se debe informar a los interesados qué es lo que se espera lograr, las acciones que se realizarán, el tiempo necesario, los indicadores que se utilizarán (pueden ser de resultados o de desempeño), la periodicidad de las reuniones para medir los cambios y la forma de comunicarlos.

Comunicar con claridad el proceso que se realizará y la forma en que cada integrante participa en este ayuda a reducir imprecisiones en la implementación. Es durante la implementación que se manifiestan con mayor fuerza las resistencias por parte de la organización; cada vez que se intente cambiar algo, la naturaleza de la empresa buscará protegerlo y mantenerlo en su condición actual.

Compromiso de líderes. Entre más personas estén convencidas de la necesidad del cambio, mayor impulso se podrá generar al iniciarlo. Los líderes de las distintas áreas son un punto fundamental para permear las estrategias y comprometer a los demás colaboradores; se debe dedicar el tiempo para desarrollar las nuevas capacidades que se requieren y facultar a dichos colaboradores para que sean agentes de cambios efectivos.

La disponibilidad para cambiar, el compromiso (actitud y energía hacia el cambio) y capacidad (conocimientos y habilidades) necesarias para realizar el esfuerzo son elementos que se deben desarrollar (Rothewll \& Sullivan, 2005). Es posible que parte de los colaboradores cuenten con alguno de ellos en mayor o menor cantidad, pero es parte del proceso de gestión apoyarlos para que los obtengan y desarrollen.

Valoración. Puestas en marcha, las distintas acciones que integran las estrategias deben ser valoradas oportunamente y de acuerdo con su naturaleza, es decir, si es una acción conductual, debemos valorar la modificación de esta, si es una acción financiera, debemos medir su resultado y, a partir de este, continuar, modificar o reforzar. Recomienda Kotter (1997) que, en caso de que se alcancen logros, aunque sean menores, estos se deben reconocer y celebrar, pues proporcionan la evidencia necesaria a los equipos para continuar con los esfuerzos e intensifican el ímpetu.

Resultados. Evaluados los resultados, se deben comunicar, cualesquiera que sean; se puede caer en la tentación de solo publicar los que son favorables, pero este tipo de pensamiento o modelo mental (Moreira, Greca \& Palmero, 2011) no aporta valor al proceso de gestión. Los resultados se deben publicar en tableros visibles a todos los colaboradores, esto permitirá conocer de primera mano los resultados que se están obteniendo y entender si el esfuerzo es suficiente o se sebe modificar; esto se revisa en juntas de alineación estratégica semanalmente. Este tipo de juntas deben ser muy ágiles y específicas.

\section{CONCLUSIÓN}

Cierre. Una de las grandes satisfacciones que da el proceso de consultoría es que es finito, tiene un final. Desde la firma del contrato se establece el periodo de trabajo que comprende. Al aproximarse la conclusión del proyecto, se debe comenzar a cerrar los temas por área y verificar que la transferencia del conocimiento y tecnología se haya realizado de forma adecuada, y que la organización pueda correr los procesos sin necesidad de los consultores; esta será la mayor prueba de que el proceso fue llevado de manera idónea.

Evidencias. Durante todo el proceso se realizaron mediciones, se modificaron estructuras, se alinearon procesos, se capacitó y facultó personal, se generaron nuevas políticas y reglamentos. Todo el trabajo realizado se debe entregar al final en una carpeta de evidencias, que servirá como memoria gráfica del proceso. 
Extensión. Algunos procesos requieren más tiempo, ya sea por cuestiones ajenas a la consultoría, por proyectos que están siendo evaluados, por resultados exitosos o continuidad del proceso de cambio; cuando se presenta este tipo de situaciones se debe llegar a un acuerdo con la organización para realizar la extensión necesaria.

Cancelación. Las causas se pueden generar desde la organización (el cliente) o desde la consultoría (el proveedor).

Atribuibles a la consultoría. Falta de resultados, desconocimiento del tema o poco involucramiento de parte del equipo de consultores.

Atribuibles a la organización. Falta de involucramiento de la dirección, no participación de líderes en las acciones, negación al cambio y falta de pagos.

Despedida. De la misma forma que se realizó una junta para presentar el proyecto y solicitar el apoyo al inicio, en sentido inverso se efectúa la junta de despedida y agradecimiento. Se reúne a las personas que participaron y se presenta el reporte final, se hacen públicas las recomendaciones necesarias, se felicita a los artífices y actores destacados del proyecto y se da por terminado.

\section{Estabilidad}

Lo que se busca en el proceso de gestión del cambio es la estabilidad de la organización, que no se debe confundir con estática. Es una etapa de balance financiero en la que se generan utilidades, se opera con eficacia y los colaboradores están satisfechos.

Este es uno de los procesos comunes y a grandes rasgos de consultoría en desarrollo organizacional y de negocios in situ, no es restrictivo y no es excluyente con otros elementos; para el caso de la presente nota metodológica, se intentó acotar a un proceso generalizado. Lo más valioso del proceso es que cumpla con los objetivos esperados y establecidos. En la figura 2 se puede observar el modelo.

Figura 2. Modelo de consultoría en desarrollo organizacional y negocios in situ

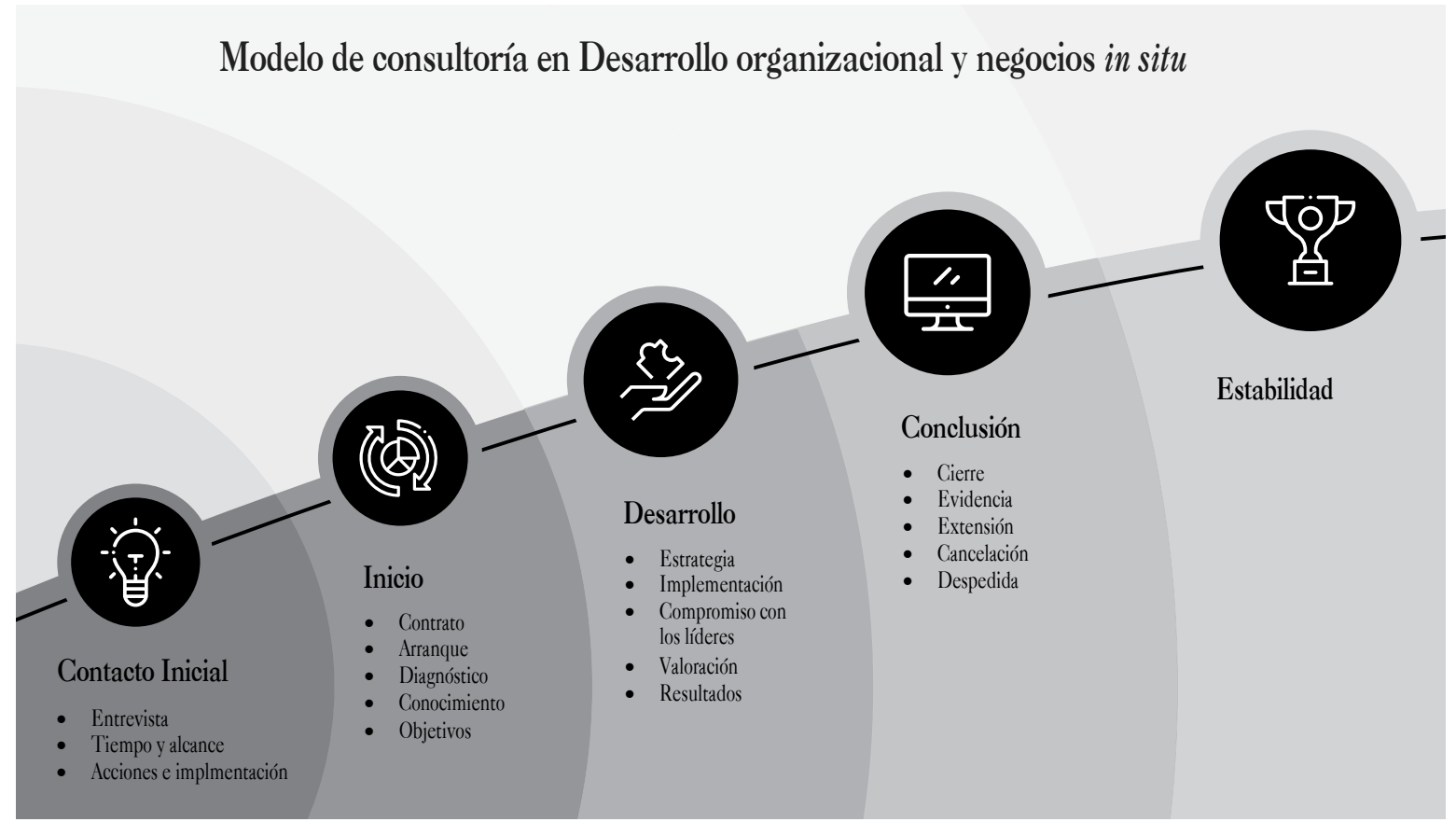

Fuente: elaboración propia. 


\section{REFERENCIAS}

Kotter, J. (1997). El lider de cambio. México, D.F.: McGraw-Hill.

Matus, D. (2018). Implementación operativa de un modelo de intervención para la mejora del desempeño organizacional. Signos, 10(2), 71-86. DOI: https:// doi.org/10.15332/s2145-1389.2018.0002.04

Moreira, M., Greca, I. y Palmero, M. L. (2011). Modelos mentales y modelos conceptuales en la enseñanza y aprendizaje de las ciencias. Revista Brasileira de Pesquisa em Educação em Ciências, 2(3). Recuperado de https://periodicos.ufmg.br/index.php/rbpec/ article/view/4134

Organización de las Naciones Unidas, ONU. (2018). Mipymes en América Latina, un frágil desempeño y nuevos desafíos para las políticas de fomento. Cepal. Recuperado de https://repositorio.cepal.org/bitstream/ handle/11362/ 44148/ 1/ S1800707_es.pdf

Rothwell, W. \& Sullivan R. (2005). Practicing Organization Development (2.a ed.). California, Estados Unidos de América: Pfeiffer, Wiley. 